\title{
\IF SHE'S TOLD YOU HER NAME IS ALICE, SHE'S LYING.« ALS GEISTERFAHRER UNTERWEGS AUF DEM LOST HIGHWAY. DAVID LYNCHS TUNNELBLICK ALS REFLEKTOR VON SELBSTLÜGE UND IDENTITÄTSSUCHE
}

\author{
Kerstin KRATOchwill Und Christine Simone Sing
}

\section{Auffahrt auf den Lost Highway}

\author{
»Funny how secrets travel [...] And the rain sets in \\ It's the angel-man. I'm deranged « ${ }^{1}$
}

Die Filme von David Lynch stellen eigene kleine Mikrokosmen im Makrokosmos der Kinolandschaft dar. Wie in einer biologischen Nische, ist jedes Werk in der Filmographie von Lynch ein komplexes, eigens zu entzifferndes Erzählwerk, das mit eigenwilliger Population bevölkert und in einer ungewöhnlichen Habitat inszeniert ist. Dabei sind diese Film-Welten nicht um sich kreisende, solipsistische Planeten, sondern besitzen durchaus sich verbindende Durchgänge und Zugänge zur Interpretation unserer eigenen ambivalenten Welt. David Lynchs enigmatische Art des Erzählens ist geprägt vom Einsatz von Leitmotiven, sei es auf inhaltlicher, optischer oder musikalischer Ebene. Diese suggestiven »Lynchismen«, wie sie immer wieder bezeichnet werden, sind ein Teil von »Lynchland«, in welches man unweigerlich gerät, auch wenn man die Auffahrt Lost Highway nimmt. Eines dieser

1 D. Bowie: »l'm deranged« (Titelsong von Lost Highway). 
immer wiederkehrenden visuellen Strukturmerkmale in Lynchs Filmen ist das der »Straße«. Allein die letzten drei seiner Filme Lost Highway (1997), Straight Story (1999) und Mulholland Drive (2001) greifen auf diese Leitmotivik zurück. Mit Lost Highway, um den sich es im Folgenden handeln soll, hat Kultregisseur David Lynch einen Thriller voller Rätsel und Geheimnisse um das Sinnbild der Straße herum geschaffen: Er übernimmt auf radikale Weise die subjektive Perspektive eines Mannes und zeigt uns, wie dieser die Welt sieht. Der Zuschauer wird auf eine ebenso erotische wie bedrohliche Fahrbahn der Lügen und Leidenschaften gezogen und ein abgründiges Spiel mit Identität und Doppelgängern, Wirklichkeit und Wahrheit nimmt seinen Lauf. Die komplexe Erzählstruktur des Filmes ist dabei nicht einfach zu entwirren, weiß man doch auf den ersten Blick nicht genau, in welche der parallelen Welten und Identitäten man gerade blickt. Auch der Drehbuchautor Barry Gifford selbst will in seiner Beschreibung des rätselhaften Handlungsablaufes keine eindeutige Lösung offerieren: »Ich halte diesen Film für eine höchst realistische, geradlinige Fallstudie eines Menschen, der mit seinem Schicksal nicht fertig wird. Doch es geht noch um vieles mehr. Jede Erklärung muss unzureichend bleiben, weil man einen Film sehen muss. $\ll^{2}$ Die Tragweite des Visuellen, auf die Gifford hier anspielt, ist einer der Schlüssel zum Rätsel des Films Lost Highway. In dem Film spielt dabei nicht nur die Straße als optische Wiederholung für die besondere Erzählstruktur des Films eine wichtige Rolle, sondern ebenso all das auf ihrem semantischen Feld herumfahrende Arsenal, wie Fahrzeuge, Scheinwerfer, Steuerräder, Mittellinien, etc.

Dieser Artikel versucht nun, ein paar der Geheimnisse, die auf oder neben diesem Highway liegen, zu ergründen. Dabei soll in der Interpretation von diesem bereits viel diskutierten Film ein Spurwechsel unternommen werden, und das Thema Selbstlüge und Identität im Scheinwerferlicht stehen. Dabei spielt der semantische Rahmen ${ }^{3}$ der

2 David Lynch in: C. Rodley (Hg.), David Lynch. Lynch über Lynch, Frankfurt/Main 1998, S. 292-293.

3 Rahmen sind bestimmte Kategorien bzw. die Versprachlichung von Denkvorstellungen, wodurch bestimmte, damit assoziativ verknüpfte Begriffe hervorgerufen werden. Vgl. C.J. Fillmore: »Frames and the 
Autobahn eine wichtige Rolle, dessen Symbolik durch allerlei filmische Gestaltungsmittel gestützt wird. In erster Linie ist dabei an die visuellen Kontraste zu denken, die im folgenden in einer Art close reading des Films erörtert werden.

\section{Cruise control auf dem Lost Highway}

\subsection{Visuelle Kontraste und Filmsemantik}

»Contrast is what makes things work. $\varkappa^{4}$

Lost Highway ist ein sorgfältig konstruierter Film, bei dem hinsichtlich der filmischen Gestaltungsmittel nichts dem Zufall überlassen wurde. Allein das Spiel mit Kontrasten transzendiert das bloß Visuelle und wird so zum sinnstiftenden Element par excellence. Auf den ersten Blick scheint der den Film konstituierende Hell-Dunkel-Kontrast, dem bekanntlich eine exponierte Stellung innerhalb des filmischen Gesamtwerks ${ }^{5}$ zukommt, auf der inhaltlichen Ebene mit der Dichotomie Gut-Böse zu korrelieren. Doch wird man auf eine Umsetzung dieser Gegenpositionen im konventionellen Sinne vergeblich warten, denn sie vereinen sich im Protagonisten Fred/Pete ${ }^{6}$ selbst: Er ist sein eigener Gegenspieler, das Gute und Böse finden in seiner (gespaltenen) Persönlichkeit den - zugegebenermaßen - klaustrophoben Raum, den er sein Leben, ja seine Identität nennt. Daher erweist sich bei näherer Betrachtung das Dichotome weitaus vielschichtiger und komplexer, denn Fred und Pete stellen nicht wirklich Gegenpositionen dar: Einzig und allein ihre Wirkung auf Renee bzw. Alice und dementsprechend ihre Sexualität konstituiert den Gegenpart, beide bleiben sich selbst

Semantics of Understanding «, in: Quaderni di Semantica: Rivista Internazionale di Semantica Teorica e Applicata 6 (1985), S. 222-254.

4 Vgl. http://www.geocities.com/ mikehartmann/de/index.html vom 21. August 2003.

5 Eine veritable Ausnahme stellt in diesem Zusammenhang sicherlich Straight Story dar.

6 Die Schreibung der Filmcharaktere basiert auf dem Original-Drehbuch von Lost Highway. Vgl. D. Lynch/B. Gifford: Lost Highway, London 1997. 
entgrenzt und der Spielball einer ebenso dominanten wie raffinierten Frau. Freilich üben diese Frauen, die Ehefrau Renee und das Traumgespinst Alice, welche ironischerweise Wakefield ${ }^{7}$ heißt, diese manipulative Persuasion auf unterschiedliche Weise aus. Der HellDunkel-Kontrast charakterisiert daher verschiedene Handlungssequenzen, denen jedoch kein einfaches Schema von Gut oder Böse zuzuordnen ist. Zunächst einmal ist man versucht, Hell-Dunkel in das Paradigma einer Licht-Finsternis-Deixis einzuordnen. Dies scheint auch die zweigeteilte Ästhetik des Films zu suggerieren. Freds Welt ist die der Schwärze, nicht umsonst taucht der dämonische Mystery Man in diesem Realitätsszenario zuerst auf, ein Dämon, dessen fahles Gesichtsrund unter künstlicher Beleuchtung an den bleichen Mond eines Gemäldes Caspar David Friedrichs erinnert. Die Ästhetik des Films zeigt zudem starke Anklänge an die Lyrik der Romantik, so auch in Goethes Willkommen und Abschied, wo die schwarze Nacht von einem bleichen Mond beleuchtet wird. Ein weiteres Motiv der Romantik, das Spiegelmotiv, wird uns weiter unten wieder begegnen. Freds kontrastloses Leben wird durch das Überblenden von Dunkel durch Schwarz und das gleitende Auflösen seiner Figur im Schwarz des Hauses unterstrichen. Dem Anschein nach absorbiert das klaustrophobe Haus das gleißende Licht, das in der Außenwelt herrscht. Am Bezeichnendsten ist die Schwärze jedoch im Gang, der zu beider Schlafzimmer führt, das man durch einen roten Vorhang erreicht ${ }^{8}$. Dieser finstere Tunnel erscheint schier unergründlich und jedes Mal, wenn Fred dort hinein läuft, verschwimmen seine Konturen, er wird allmählich unsichtbar, um sich alsbald bis zur Identitätslosigkeit aufzulösen. Bezeichnenderweise kann ihn seine Frau Renee in diesen Momenten auch nicht finden: Mehrmals wird die Sequenz dargestellt, wo Fred im Dunkel des Hauses verschwindet, während Renee im grellen Badezimmer ist und Unheilbringendes $\mathrm{zu}$ erahnen

7 Zu den sprechenden Namen zählen ferner Pete Dayton, einem weiteren Protagonisten der »hellen« Welt sowie Fred Madison, der allmählich den Verstand zu verlieren scheint.

8 Auch der rote Vorhang stellt einen intertextuellen Bezug im Werk von David Lynch dar. In seinen Filmen, so z.B. Twin Peaks, oder zuletzt in Mulholland Drive, lehnt er sich häufig an kultursemiotische Symbole an, die rot eindeutig als Signalfarbe von Gefahr ausweisen. 
scheint. Sie ruft ihm in die Finsternis ergebnislos hinterher, doch ist der Kontakt völlig abgerissen. Der kanalisierende Blick, der die Wahrnehmung auf den Eingang des Schlafzimmers bündelt und daher an die Eingangssequenz des Films im Auto erinnert, führt ins Herz der Probleme, aus denen Freds beklagenswerter Zustand abzuleiten ist. Das Schlafzimmer als Keimzelle der ehelichen Probleme taucht immer wieder auf, es ist die Quelle des Versagens, aber auch Tatort in anderer Hinsicht: Hier soll Fred, in othellesker Rage, seine Frau Renee ermordet haben, das deuten jedenfalls die Videoaufzeichnungen an, denen als visuelle Metaphern die Funktion einer Wahrheitsinstanz zukommt.

Die Montage des Films hingegen unterstreicht das oberflächliche Spiel mit den Kontrasten. Gleichsam wie das personale Erzählen in anderen Texten haben wir in Lost Highway eine seltsam involvierte Kamera, die uns Freds/Petes Sicht der Dinge zeigt. Ein wirrer Mix aus Traumcollagen, wiederkehrenden, variationistisch abgeänderten Detailaufnahmen ${ }^{9}$ hat das Gedächtnis des Protagonisten sowohl partitioniert als auch das Erinnerte transformiert. Der Betrachter ist mit Realitätssegmenten konfrontiert, die sich collagenhaft durch die zahlreichen rekurrenten Motive zusammensetzen lassen. Diese Mosaikstückchen können dann möglicherweise zu des Rätsels ${ }^{10}$ Lösung beitragen. Auf diesen Potentialis ist größter Wert zu legen, da sich von den filmischen Gestaltungsmitteln nur unvollkommene Aussagen bezüglich der Filmsemantik ableiten lassen. Die Gründe hierfür werden in den folgenden Abschnitten skizziert. Die Filmmontage ${ }^{11}$ setzt sich dialektisch aus Parallel- bzw. Assoziationsmontagen zusammen. Zum einen ermög-

9 Dies wird z.B. durch filmische Synekdochen erreicht, die die Selektivität von Freds/Petes Wahrnehmung unterstreichen. Siehe auch Kapitel 4.3 .

10 Zwar bezeichnet David Lynch Lost Highway als Genre-Mix mit Anleihen aus Horrorfilm, Film noir oder auch Thriller, doch in Wirklichkeit sei er ein Rätsel. Vgl. C. Rodley: Lynch, S. 231.

11 Statt des Terminus Montage werden auch alternativ die termini technici Cut, Schnitt oder Editing verwendet. Im Gegensatz zu letzteren suggeriert der hier präferierte Begriff Montage eben nicht ein Aussortieren von Überflüssigem, sondern unterstreicht die dynamische $\mathrm{Di}$ mension der Synthese der verschiedenen Aufnahmen. Vgl. J. Monaco: Film verstehen. Kunst, Technik, Sprache, Geschichte und Theorie des Films und der Medien, Reinbek 1995, S. 2. 
licht es die Parallelmontage, zwei oder auch mehrere Handlungsstränge aufeinander zulaufen zu lassen, um sie in einem gemeinsamen Kulminationspunkt zu vereinen. Die Einstellungen werden hierbei in der Regel parallel montiert, dabei ist es üblich, dass die zu montierenden Szenen nicht nur im Inhalt, sondern auch formal, in ihrem Aufbau und ihrer Stimmung zusammenpassen. Die verschiedenen Handlungsebenen sind dabei meist durch Symbole miteinander verknüpft. In Lost Highway werden die beiden Parallelhandlungen zwar symbolisch verknüpft, doch geschieht dies durch eine kontrastive Doppelung bzw. Spiegelung. Auch hier spielt der Hell-DunkelKontrast eine entscheidende Rolle, da er die Konstruktion dieser parallelen Universen zusätzlich polarisiert. Durch die Parallelmontage wird die Zeitachse des Films gekrümmt, wodurch das Gesetz der Chronologie außer Kraft gesetzt wird. Dies zeigt sich zum Beispiel bei der brennenden Hütte, die den Mystery Man in der Wüste beherbergt und in der Alice verschwindet. Diese brennende Hütte taucht zum ersten Mal in der Gefängnisszene auf, die der Verwandlung von Fred zu Pete unmittelbar vorausgeht. $\mathrm{Zu}$ diesem Zeitpunkt wissen wir noch gar nichts von der »Existenz« Petes. Ein weiteres Mal taucht diese brennende Hütte auf, als Pete und Alice auf dem Weg in die Wüste sind, wobei die Rauchschwaden in einer Zeitraffer-Aufnahme in die Hütte zurückgehen. Intratextuell könnte es sich hierbei um eine Verdichtung handeln, die gleichzeitig anaphorisch als auch kataphorisch auf die jeweilige Parallelhandlung verweist. Ein letztes Mal kommt diese Hütte dann im Handlungsstrang von Fred vor, als er in der Wüste mit dem Mystery Man den Mord an Mr. Eddy/Dick Laurent begeht. Interessanterweise brennt sie dann nicht. Das heißt, die Hütte ist lediglich ein filmisches Symbol, das in beiden Handlungssträngen auftaucht, insbesondere aber die lodernden Flammen, die von ihr aufsteigen, was antizipatorisch, quasi in domestizierter Form im unnatürlich laut lodernden Kaminfeuer im Hause der Madisons kontrastiv dargestellt ist. Das Feuer ist hier folglich ein Zeichen von Gefahr und Zerstörung.

Als weiteres Beispiel für den virtuosen Einsatz der Parallelmontage in Lost Highway sei eine kurze Szene aus den letzten Filmszenen angeführt. Wir befinden uns wiederum in der Wüste, der Mord an Mr. 
Eddy/Dick Laurent steht unmittelbar bevor. Der Mystery Man hat diesem so eben seine Handkamera gegeben, die einen Porno-Abend bei Andy zeigt, bei dem auch Mr. Eddy/Dick Laurent und Renee anwesend sind. Auf einmal hören wir zwei laute knallartige Geräusche, die das Videoband akustisch untermalen. Sie nehmen jedoch die folgenden beiden Pistolenschüsse vorweg, mit denen der Mystery Man den am Boden liegenden Mr. Eddy/Dick Laurent endgültig ins Jenseits befördert. Der genialste Coup der Parallelmontage ist jedoch das Aneinanderfügen von Eingangs- und Schlusssequenz des Films. Nachdem Fred Dick Laurent beseitigt hat, fährt er mit Mr. Eddys Wagen, einem schwarzen Mercedes mit reflektierenden Scheiben und nur einem Nummernschild, das sich hinten befindet, zu seinem Haus und spricht in die Gegensprechanlage: »Dick Laurent is dead.«Es ist also möglich, das erste und letzte Bild der Filmrolle aneinanderzulegen, wie dies auch beim Möbiusband der Fall ist. Zum anderen fällt neben dem Gestaltungsmittel der Parallelmontage auch der exzessive Einsatz der Assoziationsmontage ins Gewicht. Natürlich evoziert zunächst einmal jeglicher Schnitt schon eine Assoziation, die eine innere Bilderreihe heraufbeschwört, ohne sie direkt auf die Leinwand zu projizieren. Im Unterschied dazu erzeugt die Assoziationsmontage bewusst eine Assoziationskette, indem sie selektiv bestimmte Handlungssequenzen aneinanderreiht und so eine gewisse logische Sukzession und Faktizität suggeriert. In Lost Highway fällt auf, dass die Bilder inhaltlich nicht chronologisch zusammengesetzt sind, sondern dass Freds/Petes Wahrnehmung bzw. Erinnerung die Aneinanderreihung bestimmt. Durch den geschickten Einsatz dieses Montagemittels werden die Zuschauer in ihrer Wahrnehmung in dem Maße manipuliert, dass sie nicht mehr unterscheiden können, welche Elemente des Gezeigten nun tatsächlich geschehen sind und welche den Schimären des Protagonisten entstammen. Dem nicht genug, denn der wahre Film wird nicht auf der Leinwand gezeigt, sondern muss vom Rezipienten selbst montiert werden - falls möglich. Dass dies im Falle von Lost Highway eine große Herausforderung, wenn nicht gar eine unlösbare Aufgabe darstellt, liegt nicht nur auf der Hand, sondern ist an mehreren Stellen bereits angeklungen. Dies gilt in besonderer Weise für den Moment der Verwandlung, als Fred zu Pete wird. Die anachronistische 
Erzählstruktur wird durch eine phantastische Metamorphose, die sich über Zeit, Raum und Identität hinwegsetzt, komplettiert. Solche Handlungsstränge kommen im Märchen wie auch im Traum vor. Doch diese Pseudo-Gegenwelt, die uns eine heile Weltsicht vorgaukelt, negiert sich selbst, indem sie uns bald die Fragilität von Petes »Idyll« präsentiert. Die nun vorherrschende helle Farbgebung kulminiert in den kitschigen Traumlandschaften, die Pete in den für ihn glücklichsten Momenten mit Alice vor das geistige Auge rücken. Diese Impressionen intensiven Erlebens sind ebenso überzeichnet und von begrenzter Dauer wie Petes kurzes Glück mit Alice, die er dem mafiösen Mr. Eddy ausgespannt zu haben scheint. Aber diese weichgezeichneten Bilder, man denke nur an die überhöhte Darstellung des Liebesaktes vor dem Auto in der Wüste, dienen nur der scharfen Kontrastierung mit der anderen Realität: Die Sublimierung der Romantik dieser Szene löst sich in einem Crescendo schlagartig auf, wie übrigens Alice selbst, die auf Petes in wachsender Verzweiflung wiederholt geäußerten Worte »I want you « mit einem vernichtenden "You will never have me« returniert. Zurück bleibt ein verwirrter Fred, in den sich Pete durch diesen allmählichen Kontrollverlust zurückverwandelt hat. Hier laufen die beiden Stränge zusammen, denn in der Hütte wartet der Mystery Man, ein Freund von Dick Laurent und Alice, der Fred mit der Kamera einfängt. Er konfrontiert ihn mit einer anderen Realität, denn auf Freds Frage nach dem Verbleib von Alice antwortet er ihm: »Her name is not Alice. It is Renee. If she's told you her name was Alice, she is lying."

Nur vordergründig vermag die Verwandlung Freds in Pete von einer Verbesserung zeugen. Auch Petes Zimmer im Elternhaus ist ein dunkles Echo der quasi lichtfreien Zone im Madisonschen Haus oder der Gefängniszelle. Auch er ist entgrenzt und lebt in einem Umfeld der Beziehungslosigkeit. Es findet keine direkte Kommunikation statt, seine Eltern und er tauschen nur sinnentleerte Phrasen aus und wissen ebenso wenig wie er selbst, wer er ist und was in jener mysteriösen Nacht, auf die oft angespielt wird, passiert ist. Kommunikation läuft meist auf indirekte, unpersönliche Weise über das Telefon ab. Natürlich kommt Pete beim weiblichen Geschlecht zunächst besser an als Fred. Seine Beziehung zu Sheila scheint für ihn zumindest in sexueller Hinsicht befriedigender abzulaufen als der eheliche Akt im Hause der 
Madisons. Aber in verbal kommunikativer Sicht dringt auch Sheila nicht zu ihm durch, denn ihre Kontakte beschränken sich aufs Sexuelle; so beantwortet er ihre Frage »do you care about me? « mit sexuellen Handlungen. Pete wird durch die Transformation zu einem kompetenten, beliebten Draufgänger stilisiert, der einflussreiche, wenn auch kriminelle Freunde hat, allen voran den ihn protegierenden Mr. Eddy, ein Kontrast zum müden farblosen Fred wie er schärfer nicht sein könnte. Das Idyll, das in bunten Farben geschildert und mit lebhafter bis romantischer Musik unterlegt wird, wird also inhaltlich kontrapunktartig abgeschwächt, indem etwa die mit Fred konnotierte Dunkelheit immer wieder durch die Hintertür Einzug hält. Dadurch wird Petes vordergründige Virilität und Aktivität alsbald durch die Ereignisse, im Laufe derer er immer passiver und komplexbeladener wird, konterkariert. Auch seine sexuelle Potenz ist nicht von Dauer, da er von Alices übermächtiger Sexualität überfordert ist und aus seinen Minderwertigkeitskomplexen heraus sehr schnell eine eifersüchtige Obsession entwickelt. In diesem Zusammenhang setzt Lynch gerne einen Spezialfall der Assoziationsmontage ein, die sogenannte metaphorische Montage. Dabei wird die filmische Erkenntnis nicht durch narrative Elemente vermittelt, sondern durch die symbolische Gegenüberstellung von Bildern.

\subsection{Fahrgeräusche: Die Rolle der Filmmusik}

» To me, jazz is the closest thing to insanity that there is in music. ${ }^{12}$

Die Filmmusik selbst ist äußerst kontrastreich, geht sie doch über die Funktion der bloßen Untermalung hinaus. Die Musik, v.a. die Texte der einzelnen Musikstücke, sind sinnstiftend. Einerseits segmentieren sie die Handlung in einzelne Abschnitte, sorgen andererseits aber auch für deren Weiterentwicklung. Im ersten Drittel des Films setzt Lynch völlig auf eine psychologische Geräuschkulisse. Freds langes Starren in den Spiegel oder in den leeren Raum, die Handlungsarmut und Zähigkeit, die das Eheleben von Fred und Renee charakterisieren,

$12 \mathrm{Vgl}$. http://www.geocities.com/ mikehartmann/de/index.html vom 21. August 2003. 
werden akustisch durch das Spiel mit der Kognition verstärkt. Leise, schwer einzuordnende, dumpfe Geräusche fesseln mehr als laute, sie bohren sich tiefer ins Bewusstsein ein und führen zu Verunsicherung. Das Haus der Madisons scheint mit einem Soundteppich ausgelegt, der in seiner dämpfenden Wirkung jegliches Gefühl schluckt. Dieser handlungsbegleitende Ton füllt nicht nur das Haus sondern kommt pointiert in den quälend langen Pausen der ohnehin spärlichen Kommunikation zwischen Renee und Fred zum Einsatz, wodurch ihre Entfremdung auf ein unerträgliches, beklemmendes Maß zugespitzt wird. Auch Fremdgeräusche wie das Klingeln des Telefons wirken verstörend. Die Sequenz des Telefonläutens, als Fred telefonisch überprüft, ob Renee tatsächlich zu Hause ist und liest, erscheint unendlich lange und reflektiert so die erwartungsvolle Haltung Freds, die schließlich, nach langen Sekunden des Wartens enttäuscht wird. Wieder andere Geräusche, wie Freds Saxophonspiel auf der Bühne wirken übermäßig verstärkt, was den Kontrast zur sonstigen dumpfen Stille verdeutlicht. So auch das Kaminfeuer im Wohnzimmer, dessen positive Konnotation von Wärme und Behaglichkeit durch die unnatürliche akustische Verstärkung zu einem glühenden Feuerball umgewertet wird und dadurch destruktiv wirkt. Es kann kein Zweifel bestehen: Die häusliche Idylle gibt es nicht, da die Symbole, die sonst Harmonie, Schutz und Geborgenheit vermitteln sollen, eindeutig negativ kodiert sind. Einen eklatanten Gegensatz dazu bietet die Szene; in der sich Pete und Alice das erste Mal begegnen. Lou Reeds »This magic moment« dient zwar als deren perfekte Untermalung, im Gesamtkontext betrachtet ironisiert sie jedoch diesen Moment der Verklärung und ist nur als illusionäre Brechung der Geschehnisse zu verstehen. Somit bringt der Song die Naivität Petes vollkommen zum Ausdruck. An anderer Stelle, als Pete vielleicht schon die Unmöglichkeit seiner Beziehung zu Alice erahnen kann, ist der Betrachter dank des Textes von Marilyn Mansons »Apple of Sodom« einen Schritt voraus. ${ }^{13}$ Alice hat das abendliche Date mit Pete gerade abgesagt, eine Parallelszene zum oben erwähnten erfolglosen Telefonanruf Freds - bei

13 Die biblischen Anklänge, die ein Gefühl des Sündenfalls evozieren, werden durch den warnenden Refrain »|'ve got something you can never eat « verstärkt. 
beiden Szenen ist der Grund Mr. Eddy/Dick Laurent. Daraufhin trifft sich Pete, dieses Mal auf dem Motorrad fahrend, mit Sheila, einer Art Ersatzbefriedigung für Alice, was die Gestaltung der Sexszene auch deutlich macht. Der emotionale Zustand Petes/Freds klingt auch in anderen Songtexten an, so etwa in »I put a spell on you«, ein weiterer Manson-Song, der die Ereignisse in Andys Domizil musikalisch unterlegt. Auf der Leinwand wird Alice in pornographischer Aktion gezeigt, während sie sich selbst mit Andy oben im Haus amüsiert. Petes ohnehin schon geweckte Abwehrhaltung und Eifersucht auf Alices Leben wird durch diese Doppelung amplifiziert, der Songtext gewährt einen tiefen Einblick in seine Emotionen: »I put a spell on you, because you're mine, I can't stand the things that you do. No, no, no, I ain't lyin'. No. I don't care if you don't want me, 'cause I'm yours, yours, yours anyhow. Yeah, I'm yours, yours, yours. I love you. I love you. Yeah! Yeah! Yeah!« Der obsessive Charakter seiner Zuneigung könnte nicht pointierter zum Ausdruck gebracht werden. Wie das Interieur eines solchen Charakters ausgestattet ist, zeigt der folgende Blick auf eine mögliche Inspirationsquelle Lynchs. 


\section{Kurvenreiche Fahrt auf dem Lost Highway}

\subsection{M.C. Escher als Inspirationsquelle der filmischen Narrativik}

»Die ganze räumliche Welt wird zu einem stets wechselnden Konkav und Konvex-Film. ${ }^{14}$

M.C. Escher und David Lynch haben als Künstler und Schöpfer von neuen Welten viele Gemeinsamkeiten. Zum einen sind beide schwer einer bestimmten Richtung oder Schule zuzuordnen, und zum anderen erweist sich eine erstaunliche Parallelität ihrer ästhetischen Themenfelder. Das labyrinthische Haus von Fred und Renee wirkt fast wie die filmische Übersetzung eines Escher-Gemäldes. Die häufigen Auf-, und Abblendungen und die starken Kontraste, die Lynch verwendet, sind ebenfalls Stilmittel eines Escher. Es ist von daher nicht abwegig, einen Vergleich dieser beiden Künstler zu unternehmen und zu untersuchen, inwieweit die Zweidimensionalität, die Escher schon immer auf dem Papier zu sprengen versuchte, in der Dreidimensionalität der Filme von Lynch ihr Korrelat gefunden hat. Das vielschichtige Werk Eschers lässt sich grob in vier Phasen gliedern. »Die Periode der Landschaften von 1922-1937«, »Die Periode der Metamorphosen von 1937-1945«, »Die Periode der Perspektive von 1946-1956« und »Die Periode der Annäherung an die Unendlichkeit von 1956-1970«. ${ }^{15}$ Die Durchdringung von verschiedenen Landschaften und unvereinbaren Welten, Verwandlungen und Simultanwelten, das Spiel mit Perspektiven und die Grenzen des Denkens und Darstellens auszuloten sind die immerwiederkehrenden Motive in Eschers Malerei. Alltägliche und bereits abgenutzte Denkmuster sollen dabei aufgebrochen und zur Disposition gestellt werden. Solche fundamentalen Begriffe wie oben und unten, innen und außen, rechts und links, nah und fern scheinen plötzlich relativ und austauschbar zu sein. Bei Escher entdeckt der Betrachter völlig neue Konstellationen zwischen Punkten, Flächen und Räumen, die Raumstrukturen formen und die zugleich fremde und doch durch-

14 M.C. Escher, in: B. Ernst: Der Zauberspiegel des M.C. Escher, Berlin 1986, S. 84.

15 Vgl. B. Ernst: Escher, S. $22 f$. 
aus mögliche Welten heraufbeschwören. Die Frage nach Ursache und Wirkung wird hier virulent. In Eschers Werken dominieren dabei häufig starke Kontraste, und es ist vorwiegend der graphische Gegensatz von schwarz und weiß, der das Dualitätsprinzip seiner Gedankenwelt unterstützt. Die ungewohnte Umsetzung der Verschmelzung von Gegensätzen prägt sein Werk. Ebenso ist bei Lynch diese Vorliebe starker filmischer Kontraste und miteineinader verflochtener Gegensätze zu erkennen. In Lost Highway ist dies vor allem auffällig in der Doppelung der Frauenfigur Renee als dem dunklen Motiv und Alice, als dessen helle Verwandlung und Spiegelung. Escher ist, ebenso wie Lynch, nicht einem somnambulen Surrealismus zuzuordnen, der reine Traumwelten erschafft. Zwar spielen die beiden mit der Ähnlichkeit ihrer Werke zu Träumen, sie sind aber beide eher Konstrukteure unmöglich scheinender Welten. Lynch sagt dazu: »Meine Filme können Träumen zwar ähnlich sein, aber sie haben einen Anker geworfen: [...] Meine Filme müssen immer genügend Hinweise, Schlüssel oder Erklärungen liefern, damit die Zuschauer sie verstehen können und zusammen mit mir im Film bleiben. ${ }^{16}$ Lynch hat einen Weg gefunden mit Worten zu malen, dabei verbindet er die nonverbale Kunst der Malerei mit dem Medium des Films. Die Konsequenz dieser Radikalität ist sowohl bei Escher als auch bei Lynch ein intellektuelles Verwirrspiel mit Perspektiven und Dimensionen, das dem Rezipienten die Gewohnheit und Grenzen seiner Sinne vor Augen führt. Einen Perspektivwechsel, den ungewohnten Blick auf eine gewohnte Situation, möchte Lynch in seinen Werken erreichen. Er benutzt dazu oftmals das Mittel der »Grenzerfahrung «, die seine Protagonisten in Lost Highway durchleiden. Die Oberfläche einer gewöhnlichen Story - in Lost Highway ist es zunächst ein Ehedrama und ein vermeintlicher Mord - wird bei Lynch stets aufgebrochen und um eine ungewöhnliche Perspektive erweitert: in diesem Fall die Verwandlung Freds in Pete. Lynch sagt dazu: »Ich erkannte, dass man bei genauerem Hinsehen unter dieser Idylle immer rote Ameisen entdeckt. $\ll^{17}$

16 M. Bodmer/M. Rothe: »Montage aus Gesprächen mit David Lynch: »Vorhänge reizen meine Neugier««, in: Filmbulletin 211 (1997), S. 3336, hier S. 34.

17 Vgl. C. Rodley, Lynch, S. 197. 
Die Ameisen aus Lynchs Kindheit begegnen uns frappierenderweise auf dem von Escher gezeichneten Möbiusband wieder. Ähnlich diesen unendlich marschierenden Ameisen scheint sich auch die Handlung des Filmes in einer medialen Endlosschleife der Narrativik zu bewegen.

Abbildung 1: M.C. Escher: Möbiusband II, Holzstich, 1963

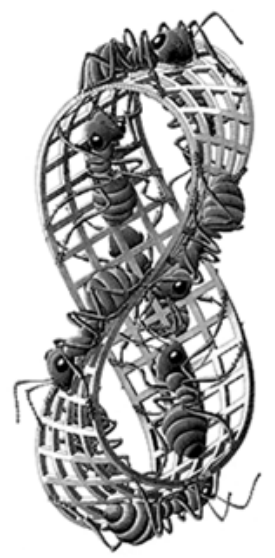

\title{
3.2 Narrative Geradlinigkeit und Gekrümmtheit am Beispiel des Möbiusbandes
}

\author{
»Dick Laurent is dead. $\ll^{18}$
}

So lauten der erste und der letzte Satz des Filmes. Es wäre daher naheliegend, die Erzählstruktur des Filmes als eine zyklische zu beschreiben, wenn da nicht der seltsame Kreuzungspunkt der Verwandlung wäre, der sich nicht so einfach auflösen lässt. Eine solche paradoxe Kreisform, die sowohl Geradlinigkeit und Gekrümmtheit in sich vereint, existiert tatsächlich: Es ist die Form des Möbiusbandes,

18 Aus der Eröffnungssequenz des Filmes, von der Lynch behauptet sie selbst erlebt zu haben: »Eines Morgens wachte ich auf, weil es klingelte, ein Mann sagte durch die Sprechanlage, [...] »Dick Laurent ist tot.« [...] Ich kenne keinen Dick Laurent«. Vgl. C. Rodley: Lynch, S. 298. 
welches nach dem Leipziger Astrophysiker August Ferdinand Möbius benannt wurde. Dieser suchte im 19. Jahrhundert nach einem Blatt Papier, das nicht zwei, sondern nur eine Seite hatte. Er fand es durch eine einfache Verdrehung. Wenn nun eine Figur - wie eben die Eschersche oder auch Lynchsche Ameise - auf diesem Streifen entlangläuft, berührt sie beide Seiten, ohne die Seite zu wechseln. Ein Punkt also, der sich auf einem Möbiusband bewegt, erscheint einem externen Beobachter, zuweilen auf der Innenseite, zuweilen auf der Außenseite, obwohl es weder Innenseite, noch Außenseite gibt. In der Zeitdimension des Films lässt sich darüberhinaus ein »Rollenswitch « zwischen »Selbstbeobachten und Selbstbeobachtetwerden« ausdifferenzieren.

Lost Highway ist ein schwer zugänglicher Film, der beispielsweise mit Douglas R. Hofstadters Buch über »Gödel, Escher, Bach« transparenter wird. In dieser Lesart erscheint Lost Highway als cineastischer Entwurf über Selbstbezüglichkeit und das endlos geflochtene Möbiusband. Hofstadter beschreibt in seinem Buch, wie die Künstler Escher und Bach, ein einziges Thema in zwei verschiedenen »Tonarten« durchspielen. Bach mittels der Fugenkomposition und Escher mit seinen bereits beschriebenen Bildern. »In einigen kann ein einzelnes Motiv auf verschiedenen Wirklichkeitsstufen auftreten. Was jedoch geschieht, wenn die Folge von Ebenen nicht linear verläuft, sondern ein Schleife bildet? Was ist dann Wirklichkeit und was Phantasie? « ${ }^{19}$ Auf die Grammatik des Filmes Lost Highway übertragen, bedeutet dies: Ist Fred/Pete real? Sind »beide« Figuren überhaupt mit den Begriffen real oder irreal zu fassen?

Dieses Paradoxon lässt sich laut Hofstadter mittels der Mathematik auflösen; und er bringt an dieser Stelle Gödel ins Spiel. Der nach ihm benannte Unvollständigkeitssatz lässt sich mit der sogenannten Epimenides- oder Lügner-Paradoxie erläutern. Epimenides war ein Kreter, der einen unsterblichen Satz aussprach: »Alle Kreter sind Lügner.« In dieser Lügenschleife sieht sich auch Fred/Pete gefangen, wenn er mit den vermeintlichen Lügen von Renee/Alice konfrontiert wird. Und diese Paradoxie stellt eine einstufige seltsame Schleife dar.

19 D.R. Hofstadter: Gödel, Escher, Bach. Ein endlos geflochtenes Band, Stuttgart 1985, S. 17. 
Solche punktuelle Situationen auf dieser Lügenschleife sind oftmals gerade mit Musik verbunden. Gerade die inneren Kämpfe Freds/Pete spielen sich immer auf diversen Partys ab oder sind mit der jeweils charakteristischen Musik umrahmt. Einer Fuge J.S. Bachs ähnelnd, geht es also einstweilen darum, ein Thema soweit zu transponieren, dass es in der jeweils neuen Gestalt eine jeweils neue Interpretation ermöglicht, bis es schließlich wieder bei der anfänglichen Form, allerdings nun auf einer höheren Stufe angelangt ist.

Lost Highway ist demnach musikalisch Bach verpflichtet und optisch M.C. Escher, indem entweder die Illusion einer endlosen Bewegung entsteht (etwa durch das Möbiusband), oder indem »die selbstreflexive Substanz die Aufhebung von Abbild und Original erreicht, etwa in der zeichnenden Hand, die eine zeichnende Hand zeichnet, die eben jene zeichnende Hand zeichnet. $\ll^{20}$ Wie eine Reminiszenz an jenes berühmte Escher-Bild erscheint hier die Szene des Filmes, in welcher der Mystery Man Fred sein Handy reicht, damit Fred mit ihm in seinem Haus telefoniere. Wie ist das möglich? ${ }^{21}$

\title{
4. Schilder auf dem Lost Highway
}

\subsection{Die Navigation durch den filmischen $(T)$ Raum}

\author{
»Die Kraft und die Schönheit des Kinos \\ bestehen jedoch in der Möglichkeit, \\ das zu erforschen, was wir nur in unserem tiefsten Innern kennen. \\ Das geht über das Freud'sche Unbewusste hinaus, \\ denn das Unbekannte kann auch außerhalb von uns liegen. « ${ }^{22}$
}

Wie obige Analyse der Erzählstruktur von Lost Highway verdeutlichte, entfaltet sich die Filmhandlung zwar anachronistisch, verfügt aber dennoch über eine gewisse Linearität. Auch für das psychische Gebilde

20 G. Seeßlen: »Ein endloses geflochtenes Band. Lost Highway von David Lynch«, in: Filmbulletin 211 (1997), S. 21-32, hier S. 32.

21 Der Originaldialog im Film lautet: Fred: »How did you do that? « Mystery Man: »Ask me«. Vgl. D. Lynch/B. Gifford: Lost Highway, S. 26.

22 M. Bodmer/M. Rothe, Montage, S. 36. 
des Traumes, so Jung ${ }^{23}$ trifft diese $»$ Kontinuität in der Diskontinuität« zu. Augenscheinlich liegt der Traum zunächst nicht in der Kontinuität der Entwicklung der Bewusstseinszustände, er droht stattdessen zum zufälligen Erlebnis zu werden, das nicht aus einer klar ersichtlichen, logischen und emotionalen Kontinuität des Erlebens hervorgeht.

Doch Jung zufolge fällt der Traum nicht vollständig aus dem bewussten Raster heraus, denn in ihm werden Ereignisse, Gedanken und Stimmungen der Vortage verarbeitet, so dass eine gewisse rückwärtsgewandte Kontinuität besteht. Gleichzeitig wirkt der Traum auch prospektiv, d.h. er hinterlässt Spuren im bewussten Geistesleben und sorgt so für mögliche Alternationen der Stimmung. Die sich selbst perpetuierenden Vor- und Rückblenden des Traumes reflektieren nicht nur die möbiusschleifenartige Erzählstruktur des Filmes, sondern erschweren auch die Deutung der Vorkommnisse auf der Leinwand. Letztlich ist nicht zu entscheiden, ob sich der ganze Film als Traum oder nur einzelne Sequenzen als traumähnlich begreifen lassen. Suggeriert die bisherige Analyse, den Großteil des Films als einen tranceähnlichen Bewusstseinszustand des Protagonisten zu verstehen, so ähnelt die Atmosphäre des Films dennoch der einer Dunstglocke. Zugegebenermaßen gibt es im Leben von Fred/Pete immer wieder luzide Momente, die sich alsbald wieder $\mathrm{zu}$ finsteren Phasen verdunkeln. Dieser changierende Aspekt zeigt zum einen die aktive Traumarbeit im Kopf des Protagonisten im Sinne einer Kompensationsleistung, zum anderen aber unterstreicht er dessen eskapistischen, ja selbstillusionären Aderlass.

Auch die Interpretation der Filmhandlung als Traum sollte dem Wechsel zwischen Wachzustand und Träumen Rechnung tragen. Denn das Geträumte ist zwar das Produkt intellektueller Anstrengung, also veritable Hirnarbeit, die Erinnerung daran, sofern sie überhaupt möglich ist, existiert nur partiell und in transformierter Version. Durch das selektive Einspeisen der geträumten Wahrnehmungen in den Bewusstseinskanal schaffen wir Intimität und Distanz zu uns selbst. Dennoch besteht, und das tritt in Lost Highway sehr deutlich zu Tage,

23 Vgl. C.G. Jung: »Die Bedeutung der Träume« in: L. Jung-Merker/E. Rüf (Hg.), C.G. Jung: Gesammelte Werke Bd. 18, Olten/Freiburg 1981, S. 201-213. 
eine ständige Interaktion zwischen Traum und Wirklichkeit. Dieser Austausch gleicht einem Teppich, dessen Fasern neu verwoben werden, wobei die »logische« Verknüpfung der im Traum auftretenden Personen, darin vorkommende Orte, die zeitliche Lokalisierung etc. in einer Art und Weise erfolgt, die Freud ${ }^{24}$ als VERDICHTUNG bezeichnet: Diese Restrukturierung erfolgt quasi ohne begreifliche $\mathrm{Zu}$ sammenhänge. So, jedenfalls, scheint es.

Bevor jedoch Funktion und Bedeutung einzelner wiederkehrender Symbole in den tranceartigen Zuständen des Protagonisten Fred/Pete analysiert werden, soll der zugrundeliegende psychoanalytische Unterbau kurz skizziert werden. Seit Sigmund Freud vor ungefähr 100 Jahren die Rolle des Unbewussten erstmals erkannt hat, gilt der Traum als Spiegel der Seele. Mehr noch als Freud stellt C.G. Jung die kompensatorische Leistung des Traums in den Vordergrund. ${ }^{25}$ Besonders interessant für die Analyse von Lost Highway ist jedoch Jungs Beschreibung des Charakters der menschlichen Seele. Jung grenzt im Laufe seiner Untersuchungen der Struktur des Unbewussten die Begriffe Psyche und Seele voneinander ab. Im Unterschied zu Psyche ${ }^{26}$ bezeichnet Seele einen bestimmten, abgegrenzten Funktionskomplex, den man am besten als eine >Persönlichkeit` charakterisieren könnte. $\mathrm{Zu}$ den gewöhnlichen Erscheinungen dieser Persönlichkeit gehören sowohl die Charakterverdoppelung als auch die Persönlichkeitsdissoziation $^{27}$. Diese Veranlagung ist zwar in jedem Menschen vorhanden, eine Pluralität von Persönlichkeiten ist jedoch in den seltensten Fällen manifest.

24 Vgl. S. Freud, Die Traumdeutung, Frankfurt/Main 1961.

25 Auf eine differenzierte Abgrenzung der unterschiedlichen Traumdeutungsansätze von Freud und Jung kann hier nicht näher eingegangen werden. Es sei nur erwähnt, dass die Ansicht Freuds, die Libido sei die einzige Quelle des Traumes, für Jung nur einen Sonderfall der Kompensationsleistung des Traumes darstellt.

26 Unter Psyche versteht Jung »die Gesamtheit aller psychischen Vorgänge, der bewussten sowohl wie der unbewussten.« C.G. Jung: Typologie, München 1990, S. 172.

27 Vgl. C.G. Jung: Typologie, S. 173: Jung geht hier noch genauer auf den Zusammenhang von Charakterspaltung und sozialer Einstellung ein, der für unsere Zwecke eine untergeordnete Rolle spielt. 
Im Unterschied dazu ist die Charakterdoppelung ein Aspekt der Anpassung an soziale Normen, wonach der Mensch nicht immer (bewusst) individuell sondern kollektiv handelt. Demgemäß verhält er sich konform zu sozialen Konventionen, täuscht sich selbst und andere über seinen wirklichen Charakter. »Er [der Mensch] nimmt eine Maske vor, von der er weiß, dass sie einerseits seinen Absichten, andererseits den Ansprüchen und Meinungen seiner Umgebung entspricht, wobei bald das eine, bald das andere Moment überwiegt. Diese Maske, nämlich die ad hoc vorgenommene Einstellung, nenne ich Persona. ${ }^{28}$

Mit dieser Bezeichnung der äußeren Erscheinung eines Menschen mit dem Wort, das die Maske des antiken Schauspielers benennt, wird auch klar, warum Jung einem Satz Nietzsches (Zur Genealogie der Moral, Zweite Abhandlung, 1887) folgend den Traum als eine innere Bühne betrachtet, auf der sich ein Schauspiel entfaltet, dessen Autoren wir selber sind. Wir sind aber auch die Regisseure und Schauspieler dieses Spiels. ${ }^{29}$ Dieser äußere Charakter, den Jung als Persona begrifflich fasst, wird durch die Anima, die Seele, komplettiert. Dabei handelt es sich nicht um das bloße Vervollständigen durch eine innere Einstellung, sondern die Jungsche Persona wird spiegelbildartig durch die Anima komplementär ergänzt. Persona und Anima sind also Schattenbilder, die menschliche Seele verfügt über einen Komplementärcharakter. Doch dem nicht genug: Diese spiegelbildliche Doppelung findet sich auch auf der Ebene des Unbewussten wieder: Nicht nur, dass sich Persona und Anima, das Außen und Innen, komplementär ergänzen, der Spiegelreflex bezieht sich auch auf den Geschlechtscharakter und ist in einem jeden Individuum angelegt. So hat jede Frau eine »männliche Seele«, die dann auch als animus bezeichnet wird, die weibliche Seele des Mannes dementsprechend als anima. Konkret bedeutet das, dass die anima das personifizierte Selbstbild der »weiblichen« Möglichkeiten und Erfahrungen des Mannes und somit einen Gegenentwurf zum >männlichen< Selbstbild der Persona darstellt. In Lost Highway finden wir diese spiegelnde Doppelung der menschlichen Seele so perfekt inszeniert vor, als wäre Jungs Typologie die

28 Vgl. ebd., S. 174.

29 Darum nennt Jung eine Deutung, die von dieser Einsicht ausgeht, die Deutung auf der Subjektstufe. Vgl. C.G. Jung: Traum und Traumdeutung, München 1990. 
Lieblingslektüre von Lynch zur Entstehungszeit des Films gewesen. Dem dunklen Fred wird eine ebenso dunkle wie passive Anima Renee ist nicht zufällig dunkelhaarig - an die Seite gestellt.

Wie aber lässt sich die Traumbedeutung ermitteln? Basierend auf den frühen Daten von Freud und Jung hat sich ein Kanon von sogenannten Traumsymbolen herausgebildet, von denen einige auch für Lost Highway fruchtbar gemacht werden können, allen voran die Symbolik des Hauses und der Fortbewegungsmittel. ${ }^{30}$ Das Haus der Madisons spielt eine zentrale Rolle im Handlungsablauf des Films. Übereinstimmend wird das Haus in der einschlägigen Literatur als Symbol des Selbst bezeichnet. So erweckt Fred wann immer er im Haus umherläuft den Eindruck von Orientierungslosigkeit. Er verschwindet wiederholt in einem dunklen Gang, steigt eine Treppe hinauf, die auf einen roten Vorhang zuführt, in der Hoffnung Verborgenes zu entdecken. Jedenfalls ist die hochgradige Rekurrenz dieser Irrwege ein sicheres Indiz seiner Identitätssuche. Immer wieder führt Lynch uns die Szene des Treppenaufgangs vor Augen, der aller Voraussicht zum Schlafzimmer führt. Den einzelnen Räumen des Hauses kommt ohnehin besondere symbolische Bedeutung.

Der Keller ist zum Beispiel ein Symbol für den Triebbereich, unsere sexuellen wie auch aggressiven Impulse. Kein Wunder also, dass Freds Übungsraum, der zudem schalldicht ist, im Keller des Hauses ist. ${ }^{31}$ Freds Sexualität und stille, latente Aggressivität, die in seinem Unbewussten rumoren, lassen sich nicht externalisieren: Der schalldichte $»$ Keller « lässt nichts nach außen dringen, worauf der psychische Druck stetig ansteigt. Interessanterweise scheint es sich bei diesem Übungsraum um denselben Raum zu handeln, der beim Hausbesuch der Polizisten als zweites Schlafzimmer bezeichnet wird. Paart man nun Freds problematische Sexualität mit der Symbolik des Saxophons, die zumeist die männliche Sexualität veranschaulicht,

30 Die folgenden Quellen bieten die Basis für die Beschreibung und Deutung der Traumsymbole: E. Aeppli: Der Traum und seine Deutung, Stuttgart 1967; H.B. Flöttmann: Träume zeigen neue Wege, Stuttgart 1998.

31 Die Geographie des Hauses bleibt allerdings unklar, was ein weiteres Indiz dafür sein könnte, dass Fred sich in seinem Selbst nicht zurechtfindet. 
lassen sich weitere Analogien zwischen dem Sinnbild des Kellers, des Schlafzimmers und des Musikinstruments ziehen. Spielt der sonst so introvertierte Fred auf seinem Saxophon wirkt er seltsam verändert: Er scheint aus sich herauszugehen, doch gemessen an der Wirkung auf seine Frau, die beträchtliches Desinteresse an seinem Spiel zeigt einmal verlässt sie den Club mit Andy, ein anderes Mal geht sie erst gar nicht mit - symbolisiert das Saxophon wohl eher die gestörte Kommunikation der Ehepartner als ein erfolgreiches Ventil für männliche Sexualität. Das wird auch dadurch verdeutlicht, dass erfüllende sexuelle Handlungen gerade nicht im eigenen Schlafzimmer, sondern außerhalb des Hauses stattfinden, sei es im Auto oder im Hotelzimmer und - von besonderer Brisanz - im Lost Highway Hotel.

Die oben beschriebene Symbolik des Hauses und seiner Räume erfährt eine Amplifikation in Gestalt der dort herrschenden Lichtverhältnisse. Das dominierende Dunkel fungiert selbst als Symbol für das Unbewusste, was den Somnambulismus, mit dem Fred durch die Nacht des Hauses schleicht, aufs Trefflichste beschreibt. Aber auch sein Gegenpart die Helligkeit, die für das Bewusstsein steht, leuchtet hin und wieder im Dunkel des Hauses auf. Doch die spärlichen Lichtquellen sind in der Mehrzahl artifiziell. Selbst die Fenster scheinen das Sonnenlicht von draußen zu reflektieren statt zu brechen, was das gestörte Verhältnis Freds zu seiner Außenwelt indiziert. Die wenigen Lampen sorgen für eine punktuelle Akzentuierung während natürliche Lichtquellen wie das Kaminfeuer und die Zigarettenglut, die am Anfang des Films das erste Licht auf den Protagonisten wirft, akustisch verfremdet werden. Diese erdrückende Omnipräsenz des dunklen Unbewussten und seiner Zwänge überschattet allmählich Freds ganzes Leben und macht ihn wahnsinnig.

Weiterhin spielt das Feuer eine wichtige Rolle unter den Traumsymbolen. Als Wandlungssymbol steht es für große seelische Gewalten, die meist einen destruktiven, wenn nicht sogar selbst zerstörerischen Verlauf nehmen. Behandelt der Traum gar einen Brand im Dachgeschoß, so ist die Analogie zu schweren geistigen Störungen naheliegend. Eine Variation des Madisonschen Hauses ist die Hütte in der Wüste, bei welcher der Dachstuhl in Brand geraten ist und die außer den Sofas der Madisons nichts enthält. Die Hütte ist eine 
veritable Kopfgeburt Freds, die eben darum auch den Mystery Man beherbergt und am Schluss Alice Asyl gewährt.

Die Tatsache, dass sich Fred mental sehr beengt und angegriffen fühlt, wird auch dann deutlich, als die Polizisten nach dem Auftauchen der Videobänder sein Haus durchsuchen. Ein Polizist steigt auf das Dach des Hauses und wirft einen Blick nach innen, eine Sache, die Fred nicht goutiert. Es liegt auf der Hand, dass er sich beobachtet fühlt. ${ }^{32}$ Eine auch nur potentielle Introspektion seines Seelenlebens erfährt er als quasi existenzielle Bedrohung. Dem nicht genug: Da es sich bei den Polizisten um Autoritätspersonen handelt, die das Über-Ich repräsentieren könnten, fühlt sich Fred wohl doppelt kontrolliert und seines Schutzes beraubt. Das zeigt sich deutlich in der Szene, in welcher die Dachfenster, die sich anscheinend über dem Bett befinden und eine angenehme Lichtquelle sein könnten, als zudringlich empfunden werden. Überhaupt ist das Schlafzimmer bei Lichte betrachtet kaum wiederzuerkennen. Wird es bei Dunkelheit als gefährlicher Ort empfunden, wirkt es bei Helligkeit eher kalt als riskant. Das lässt sich vom dunklen Zimmer Petes nicht behaupten, das kalte Licht seiner Deckenleuchte, die an die Lampe der Gefängniszelle erinnert, zieht allerlei Ungeziefer an. Es wimmelt nur so von Insekten, die seinen Gemütszustand illustrieren. Auch sie können für (sexuell kodierte) Zudringlichkeit stehen. Das massenhafte Auftreten von Insekten ist als Gefahrensymbol zu werten, andererseits auch als Zeichen des angegriffenen Nervensystems. Die rastlosen Ameisen aus Eschers Zeichnung des Möbiusbandes scheinen in Petes Nervenbahnen gekrochen zu sein. Und so spiegelt auch das Verhalten Petes, der gerade von Alice einen Korb für das abendliche Date erhalten hat, diesen Angriff auf das Nervensystem wider, einen Übergriff, den man im wahrsten Sinne des Wortes im Kopf nicht aushalten kann.

Die deutlichste und konkreteste Warnung geht jedoch von der Spinne aus, die sich langsam an der Wand von Petes Zimmer hinaufbewegt und in Großaufnahme gezeigt wird. Hier setzt Lynch auf das Spiel der Effekte von hochgradig stereotypen Symbolen. Die Spinne ist ein archetypisches, mythologisiertes Angstsymbol, das immer wieder bemüht wird. Fast immer bringt die Spinne Verderben, zieht an den

32 Das Haus der Madisons liegt zudem noch unterhalb der Sternwarte. 
Fäden des Schicksals wie die Dämonin einer unergründlich fremden Welt. Als tiefenpsychologisches Traumsymbol drückt sie vorbewußte Inhalte aus, sozusagen ein Dämmern dessen, was einem als Schicksal begegnen könnte. Und natürlich symbolisiert Arachne die Abgründe der weiblichen Seele, der man ins Netz gehen kann. In diesem Fall ist das Schicksal des jungen Pete durch diese Spinne besiegelt, die teuflische, obwohl blonde Alice hat ihn schon vollkommen umgarnt.

Da verspricht auch Petes stürmische Fahrt auf dem Motorrad keine Besserung, die unmittelbar auf diese insektenreiche Szene folgt und geradewegs zur nächsten Sexszene im Hotel führt. ${ }^{33}$ Der todbringende Schwan überblickt das Geschehen, wodurch sich die Anzeichen des imminenten Kulminationspunktes verdichten. Der Übergang zum nächsten Entwicklungsstadium in Freds/Petes schleichendem Zerfall wird durch das Motorrad als Schwellensymbol eingeläutet. Das Motorrad ist ein Zeichen für stürmische Individualität, die jedoch von Pete nicht einmal im Ansatz erreicht wird. ${ }^{34}$ Im sonst sehr von hochmotorisierten Automobilen dominierten Werkstatt- bzw. Halbweltmilieu, in dem sich Fred/Pete bewegen, kann diese exzeptionelle Fahrt auf dem Motorrad höchstens als gewagter Schritt, als Versuch der Individuation, gewertet werden. Die schnellen, sehr gepflegten Autos im Film repräsentieren das Äußere des Fahrers sowie seine geistigseelische und körperliche Potenz. Die Fahrt mit dem Auto veranschaulicht allerdings auch den Lebensweg bzw. die Lebensbewältigung. Dabei ist es entscheidend, ob man das Auto selbst lenkt oder ob man als Beifahrer oder gar auf dem Rücksitz des Autos mitfährt. Es fällt auf, dass Fred eigentlich immer selbst fährt, während Pete zwei Mal gefahren wird. Einmal überläßt er Mr. Eddy das Steuer, ein weiteres Mal fährt er im Bus zum Haus von Andy, wo Alice auf ihn wartet. Beim ersten Fall verläuft die Fahrt auch sehr rasant, Mr. Eddy

33 Statt der begehrten Alice projiziert Pete nun seine Wünsche auf Sheila, sein Blick geht folglich auch an Sheila vorbei direkt in die Kamera, in die er nach links unten wie in einen Spiegel blickt.

34 Freds Ventil ist sein Job als Musiker. Es ist auch kein Zufall, dass Lynch ihn das Saxophon, dem ein verrucht-sinnliches Konnotat anhaftet, spielen lässt. Das ist der einzige Moment, in dem der sonst introvertierte Melancholiker einen aktiven Part übernimmt. Doch diese Ausbruchsversuche sind nur von kurzer Dauer und zum Scheitern verurteilt. 
demonstriert sehr deutlich seine Überlegenheit und Macht. Und im menschenleeren Bus wird Petes einsetzender Kontrollverlust betont, der Bus steht zwar für Zuverlässigkeit, doch scheint Petes Kontakt zur Außenwelt gestört, denn er sieht nur sich selbst im Spiegelbild der Scheibe. Alle Arten von Fortbewegungsmitteln dienen per definitionem der Überbrückung von Distanzen und fungieren somit als Schwellensymbole. In der klassischen Definition wird so die Überleitung von einer Traumphase in den wachen Zustand bewerkstelligt. In Lost Highway wird damit eher dem Auto als Sexualsymbol bzw. das Springen auf der Zeitachse bzw. einigende Verbindung von Handlungssegmenten Rechnung getragen. Das Auto hat demnach zum einen handlungsbefördernde Wirkung und transportiert so die Handlung durch das Dahingleiten im Auto weiter. Zum anderen symbolisiert es die fehlende Verankerung von Fred/Pete in sich selbst sowie in der ihn umgebenden Gesellschaft.

\subsection{Der Blick in den Spiegel}

»People are going to have to get used to different types of focus. ${ }^{35}$

Zum einen ist das Spiegelmotiv ein bedeutender Topos in der Literatur, zum anderen aber auch ein signifikantes Traumsymbol. Die Redewendung »der Spiegel der Wahrheit« verweist bereits auf seine wesentlichste Bedeutung: der Spiegel dient der Wahrheitsfindung, Reflektieren heißt, nachdenken, vor allem über sich selbst. Der Blick in den Spiegel heißt auch sich selbst erkennen, seine Stärken und Schwächen sehen, sein Wirken auf andere wahrzunehmen. ${ }^{36}$ Auch Aeppli ${ }^{37}$ argumentiert dahingehend, dass das häufige Vorkommen des Spiegels auf die Suche des Träumers nach sich selbst hindeutet; so versinnbildliche der Spiegel den Intellekt, der das Zerrbild des Selbst wieder zurecht rücken und somit die eigene Identität wiederherstellen solle.

$35 \mathrm{Vgl}$. http://www.geocities.com/ mikehartmann/de/index.html vom 21. August 2003.

36 Vgl. H.B. Flöttmann: Träume, S. 182.

37 Vgl. E. Aeppli: Traum, S. 332. 
Der Spiegel als Symbol von Erkenntnis weist eine lange Tradition auf, die früh in Innenperspektive und Außenperspektive differenziert. ${ }^{38}$ So unterscheidet Augustinus in seiner Theorie des Sehens zwischen dem Bild im Auge und dem in der Seele. Während letztere ein Bild aktiv formt, ist das Sinnesorgan ein bloßer Reflektor, der die Bilder der Seele passiv widerspiegelt und sie auch nicht festhalten kann. Somit erfährt das Auge die Charakterisierung eines toten, stofflichen Spiegels. Die Maxime »das Auge als Spiegel der Seele« beruht auf ebendiesem Verständnis. Besteht eine Harmonie im Sinne eines ausgesöhnten Innen und Außen, ist das Auge klar, daher gilt das ungetrübte Auge als Metapher der Wahrheit. Mit der neuzeitlichen Entwicklung der Individualität erhält der Spiegel natürlich zusätzliche Signifikanz, welche die bei Augustinus angedeutete Zweiteilung in Innen und Außen um eine weitere Dimension verschärft: die Kluft zwischen dem inneren Raum als persönlichem Refugium und dem äußeren, sozialen Eingebundensein. Durch dieses neue Verständnis von Individualität und Selbst kommt es zum Auseinanderfallen eines »wahren« Ichs und seiner gesellschaftlichen Rollen. Der Blick in den Spiegel ist somit ein domestizierter Blick, der sich den Spielregeln gesellschaftlich geregelter Selbstbeziehung unterwirft und die Innerlichkeit als $\mathrm{Zu}-$ fluchtsort des ganzheitlichen Menschen als Utopie hinter sich lässt. ${ }^{39}$ Die Entfremdung des Menschen von sich selbst könnte nicht pointierter dargestellt werden als in Lost Highway. Die idealistische Sicht des Menschen als einem Ganzen bestehend aus komplementären Hälften wird durch die Persönlichkeitsdissoziation Freds/Petes und die daraus resultierende Doppelung auf drastische Weise zugespitzt. Der Blick in den Spiegel liefert keine Antworten mehr, er wirft lediglich viele neue Fragen auf.

Somit wird der Spiegel in Lost Highway zu einer doppelten Gefahr. Zum einen zeigt der verzweifelt fragende Blick Freds in den häuslichen Spiegel, dass es längst zu einer Entgrenzung von ihm und der Welt gekommen ist. Er kann nicht einmal zu sich selbst eine Verbindung herstellen, es sei denn in die Abgründe seiner Seele. So ist denn auch

38 Vgl. E. Peez: Die Macht der Spiegel. Das Spiegelmotiv in Literatur und Ästhetik des Zeitalters von Klassik und Romantik, Frankfurt/Main 1989.

39 Vgl. L. Trilling: Sincerity and Authenticity, London 1972. 
eher der Mystery Man als Reflex seiner selbst zu sehen als das Bild, das Fred im Spiegel erhält, mit dem er aber nichts anzufangen weiß. Im Gegensatz dazu konvertieren andere Flächen, wie die Fensterscheibe oder das Busfenster, zu unfreiwilligen Spiegelflächen, die den Protagonisten nachgerade zur Reflexion zwingen wollen. Die Spiegelsymbolik ist in Lost Highway auch in anderer Hinsicht von Bedeutung, nämlich im Motiv der Spiegelung in den Augen des Geliebten. Dabei vereint der Spiegel gleich zwei Reflexe: Ein jeder sieht sich selbst und seine Augen spiegeln das Innere wider; der jeweils andere beschaut in den Augen des Geliebten sich selbst in einem Spiegel. Eines Nachts sieht Fred, nachdem er sich im Schlaf gewälzt hat, im Gesicht von Renee den Mystery Man als Reflex seiner selbst. Letzterer verkörpert die dunkle Seite seiner Seele, die Abgründe des Spießers. Auch der Bourgeois begehrt die Orgie, trotz oder gerade wegen seiner Entgrenztheit von sich Selbst, die ihm in seiner Ich-Auflösung durch Anpassung widerfährt. An anderer Stelle soll der Blick in die Augen Renees wieder zu einem komplettierenden Reflex führen, doch interessanterweise ist in ihren abgeschirmten Augen nichts zu entdecken, so sehr sich Fred auch bemüht, darin zu lesen, bevor sie den Liebesakt vollziehen. Der Blick bleibt ohne Reflex, statt dessen scheint Fred in die Kamera zu blicken, die ihn von rechts unterhalb des Bettes aufnimmt. Dies ist eine Parallele zu oben erwähnter Sexszene von Pete und Sheila, wo der Blick in die Augen auch nicht stattfindet und die Kamera die Verbindungslosigkeit einfängt.

Eine weitere Schlüsselszene, in der die Spiegelsymbolik im Film zum Einsatz kommt, ist auch ein entscheidender Moment im Leben des jungen Pete. Er fährt gerade im Bus zu Andys Haus, wo Alice auf ihn wartet. Pete sind längst Zweifel an seiner Liaison dangereuse gekommen. Die Kamera wandert hier von Petes seitlichem Spiegelprofil im Busfenster zu Pete selbst. Dieser verkehrte Blickwinkel erlaubt einen tiefen Einblick in seinen seelischen Zustand: sein Spiegelbild ist realer als er selbst. Interessanterweise ist er wiederum im Profil aufgenommen, was das janusköpfige Wesen seiner Existenz ${ }^{40}$ verdeutlicht.

40 Dies zeigt sich auch an der Wunde, die Fred/Pete kurz vor bzw. kurz nach der Metamorphose haben; während Fred einen roten Fleck auf der linken Stirnseite trägt, ist dieser bei Pete auf der rechten. 
Der einzig positive Kontext des Spiegelmotivs ist die erste Begegnung von Alice und Pete. In dieser Szene, die in der Werkstatt, einem Ort der Kreativität, stattfindet, herrscht eine helle Klarheit, die einen direkten Blick in die Augen zulässt. Die Magie des Spiegels wird auf musikalischer Ebene mit Lou Reeds »This magic moment« perfekt unterlegt. Und schließlich fungiert der Spiegel auch als ein Art visueller Metapher. Während Mr. Eddys rasanter Fahrt auf dem Highway sitzt Pete auf dem Beifahrersitz. Mehrmals wirft Mr. Eddy einen Blick in den Rückspiegel, mehrmals dreht Pete sich um. Diese Retrospektive führt zu interessanten Beobachtungen, denn bald scheint das nachfolgende Auto ohne Fahrer, bald scheint der Fahrer an Fred zu erinnern. Petes Blick zurück in die Vergangenheit scheint ebenso wenig zu gelingen wie Mr. Eddys Versuch herauszubekommen, wer ihm da eigentlich auf den Fersen ist. Der Blick zurück wirft seine Schatten auf die Zukunft voraus.

\subsection{Visuelle Metaphern als Zeichen der Wahrheit?}

»I like making films because I like to go into another world. I like to get lost into another world. ${ }^{41}$

In einem Film, der die Wahrnehmung der Zuschauer in dem Maße manipuliert wie es David Lynch in Lost Highway perfektioniert hat, spielt das Auge als Sinnesorgan eine zentrale Rolle. Dem Auge wie auch anderen visuellen Metaphern muss also besondere Aufmerksamkeit gewidmet werden. Unter dem Begriff »visuelle Metapher« soll hier der symbolische Einsatz filmischer Mittel, wie beispielsweise Kameras, Filmausschnitte oder Bilder, verstanden werden, quasi ein Film im Film. In den folgenden Ausführungen soll nun zuerst die Rolle des Auges deutlich herausgestellt werden. Im zweiten Teil soll anhand anderer visueller Metaphern eine mögliche Deutung der Geschehnisse auf der Leinwand erfolgen.

Das Auge wird in zahlreichen Szenen fokussiert dargestellt. Dies ist vor allem in derjenigen Handlungssequenz der Fall, in deren Mit-

41 Vgl. http://www.geocities.com/ mikehartmann/de/index.html vom 21. August 2003. 
telpunkt Pete und Alice stehen. Mittels einer filmischen Synekdoche ${ }^{42}$ wird zunächst das Auge von Alice gezeigt als sie mit Pete telefoniert, um eine weitere Verabredung zu vereinbaren. Erst langsam gleitet die Kamera über ihr Gesicht zu ihrem Mund hinab. Im Kontrast dazu wird in der Parallelszene, als sie wieder telefonieren, ausschließlich ihr Mund gezeigt. Bringt man beide Szenen in den Gesamtkontext der Filmsemantik, lässt sich ableiten, dass Petes selektive Wahrnehmung auch visuell fragmentarisiert wird, indem zwei Bereiche von Alices Gesicht hervorgehoben werden. Als sie das Date absagt, wird nur ihr Mund gezeigt, sie verweigert ihm also einen tieferen Einblick in ihre Beweggründe. Beim zweiten Anruf aber fokussiert die Kamera ihre Augen, sie stellt eine oberflächliche Verbindlichkeit her; dadurch aber, dass sie nicht auf den Augen verweilt sondern zum Mund weiter wandert, wird diese Intimität konterkariert: dieser Mund war schon einmal die Quelle von Unwahrheit. Abgesehen von der Bedeutung des Auges spielen Videobänder bzw. die Videokamera als visuellen Metaphern eine wichtige Rolle. Sie dürften als Wahrnehmungsregulativ wenn nicht gar als Wahrheitsinstanz gelten. Nehmen wir die Filmhandlung im Grunde einzig und allein aus der Sicht des gespaltenen und womöglich auch doppelzüngigen Protagonisten Fred/Pete wahr, liefern uns die Videobänder die Möglichkeit der kontrastiven Wahrnehmungsperspektive. Diese Videobänder verbreiten eine frostige, zutiefst verunsichernde Leere seitens des Zuschauers. Ein unsichtbarer Dritter scheint im Haus der Madisons sein Unwesen zu treiben; wie ein ungebetener Gast scheint er vielmehr dort zu residieren. Allerdings zeigen die Videoaufnahmen zunächst nur das, was wir ohnehin wissen: die Sterilität des Hauses. Doch auf den späteren Bändern bekommen wir - wenn auch nur andeutungsweise - den Mord an Renee zu sehen. Wir können eigentlich gar nicht sicher sein, ob er tatsächlich passiert ist. Unser vages Wissen basiert nur auf den Aufnahmen und der verworrenen Geschichte, die sich Fred zurechtkonstruiert.

Diese Videobänder sind wohl das filmische Produkt des Mystery Man. Folgen wir der Annahme, dass dieser die Abgründe der Seele Freds repräsentiert, können wir zumindest ableiten, dass das Gezeigte

42 Vgl. J. Monaco: Film, S. 168. 
die Ereignisse aus der Sicht von Fred/Pete relativiert, wenn nicht sogar seine traumhafte Lügenwelt dekonstruiert. Die Videoaufnahmen sind frei von der selbstillusionären narrativen Ausgestaltung Freds. In dieser bereinigten, essentiellen Version lassen sich die Gegebenheiten häppchenweise auf Folgendes reduzieren. Das erste Tape stellt die akzeptable Fassade des Madisonschen Heimes dar, in dem es zwar unter der Oberfläche gewaltig rumort, wovon aber (noch) nichts nach außen dringt. Auf der nächsten Kassette werden das Wohnzimmer, insbesondere aber die Sofas gefilmt, einem sozialen Ort der domestischen Kommunikation, wo indes eine unerträgliche Leere herrscht, die mit der leidenschaftslosen Indifferenz des Ehelebens einhergeht. Die Madisons reagieren schockiert und verständnislos, als sie auf eben jenem Sofa sitzend mit dieser Gefühlskälte auf den Videobändern konfrontiert werden. Der Verdacht eines Voyeurs wird allerdings durch die dritte Kassette ad absurdum geführt, welche die beiden friedlich schlummernd im Schlafzimmer zeigt. Nichtsdestotrotz sprechen die beiden Cops das heikle Thema »Schlafzimmer« mehrere Male an. Sie wollen peinlichst genau wissen, ob Fred und Renee auch beide in diesem Schlafzimmer schlafen und ob es vielleicht noch ein weiteres Schlafzimmer gebe. Bezeichnenderweise findet Fred selbst das dritte Tape im Wohnzimmer, während Renee die anderen beiden vor der Tür fand. Es zeigt den Aufgang zum Schlafzimmer und stoppt abrupt, wie auch seine Erinnerung. Der nächste Ausschnitt aus dem Video zeigt dann Fred, wie er über die blutende Renee gebeugt ist, seine Hände sind ebenso blutverschmiert. Wir können erahnen, dass er sie aus Eifersucht umgebracht hat. Auch der nächste Mord in Freds Leben wird auf der Videokamera festgehalten. Dieses Mal aber wissen wir bereits, dass dieser Mord noch geschehen wird, das wird uns ja eingangs schon durch die Sprechanlage mitgeteilt. Aber auch da haben wir ein Korrektiv, das uns mit Zweifel erfüllt: Andy, ein Freund von Dick Laurent, weiß noch gar nichts von seinem Tod, d.h. auch hier können wir uns nicht sicher sein, ob es sich nur um ein Wunschdenken seitens Fred handelt, weil er seine Rachegelüste befriedigen will. Ein weiteres Indiz dafür ist die tatkräftige Mithilfe des Mystery Man, der Fred im entscheidenden Moment das Messer unterschiebt. Es ist auch der Mystery Man, der Mr. Eddy/Dick Laurent nicht nur den Grund für 
seine Ermordung zeigt, sondern auch sein Vollstrecker wird. Seine Videoaufnahmen zeigen Mr. Eddy/Dick Laurent in Begleitung von Renee auf einer orgiastischen Party im Hause von Andy. Abschließend soll noch auf zwei weitere visuelle Metaphern kurz eingegangen werden. Als die Polizisten das Haus des ermordeten Andy durchsuchen, stoßen sie auf ein Photo, das drei Personen darstellt: Dick Laurent, Renee und Andy selbst. Das ist die Wahrnehmung der Cops, die wir als Zuschauer teilen sollen. Pete nimmt jedoch vier Personen auf dem Bild wahr: neben den beiden Männern befinden sich auch zwei Frauen, Renee und Alice auf dem Bild. In der gleichen Szenerie betrachtet Pete mit Entsetzen den pornographischen Film unter der Mitwirkung von Alice, die schonungslos den letzten Rest seines Idylls zerstört. Auch hier fungieren diese Darstellungen auf der Videoleinwand als Korrektiv der idealisierten Sicht, mit der Pete Alice anfangs betrachtet hat; sie offerieren also ein objektivierteres Bild der Ereignisse.

Die Rolle des Videos als Wahrheitsinstanz ist natürlich auch differenziert zu betrachten. Vor allem im letzten Drittel des Films wird die Aussagekraft durch die Ko-Präsenz des Videos und des Mystery Man geschwächt. Dadurch dass alle an einem Ort vereint sind, fließen die verschiedenen Instanzen ineinander. Der Mystery Man verschwindet ebenso wie Alice. Neben der Präsentation der Quintessenz der Ereignisse, bietet das Video eine Art Außenperspektive der Filmhandlung und fungiert so als Meta-Ebene, die einen referentiellen Anknüpfungspunkt darstellt, wenn es um die Lokalisierung der Identität Freds/Petes geht. Die Selbstreferentialität, die durch den Einsatz dieser visuellen Metaphern generiert wird, verweist auf den Vermittlungscharakter des Films und wirft die Frage nach der Authentizität von Filmen überhaupt auf. Ist es möglich, die Wahrheit bzw. Realität in der Traumindustrie Film darzustellen? Sollte es in Lost Highway eine Wahrnehmungsperspektive geben, die mehr als die anderen auf eine authentische Darstellung der Dinge achtet, dann kommt diese Rolle ironischerweise den Videokameras, ganz allgemein betrachtet jeglichen Schwarzweißbildern zu. Die Wahrheit, die vielleicht auf diese Weise ans Licht kommt, sollte für Fred/Pete eine Möglichkeit zur Flucht aus der Selbstlüge sein. 


\title{
5. Geisterfahrer auf der Überholspur? Der Blick in den Rückspiegel
}

\subsection{Erinnerung und (Selbst-)Wahrnehmung: Erinnerungskonzepte}

\author{
»Eine akkurate Erinnerung an die Vergangenheit \\ würde wahrscheinlich in die Depression führen. ${ }^{43}$
}

In Lost Highway erklärt Fred den beiden Polizisten, warum er keine Videokamera habe. Er sagt, er erinnere sich gerne auf seine Art: So wie er die Dinge in Erinnerung habe, und nicht so, wie sie sich ereigneten. Dies bedeutet, dass eine »Fiktion des Ich « wesentlich wahrhaftiger für einen selbst sein kann, als die reine Tatsachenwahrheit. Bis zu einem gewissen Grad kann man - nach dieser These - jemandem seine Erinnerungen stehlen, indem man sein Leben einfach abfilmt. Lynch selbst sagt zu diesem Thema:

»Ich dachte etwa an Kindheitserinnerungen. Sie werden zu ganz besonderen Erinnerungen. Wenn Ihnen jemand nun ein Video von einem bestimmten Moment zeigte, den Sie mit sich herumtragen und an den Sie sich erinnern, würde es Sie vermutlich fast umbringen, denn es wäre so profan, es würde nur die Oberfläche des Ereignisses einfangen. Sie haben so viel hinzugefügt, dass es das Video zu einer Lüge macht und Ihre Erinnerung viel wahrhaftiger ist. ${ }^{44}$

Die geheimnisvollen und anonymen Videotapes, die Fred und Renee immer wieder vor ihrer Haustüre finden, funktionieren demnach als visuelle Metaphern für das Erinnerungsvermögen und als eine Art Tatsachenwahrheit. Was Fred und Renee hier zu sehen bekommen sind die kalten und unpoetischen Aufzeichnungen einer/ihrer völlig sinnentleerten Beziehung, die man als die »nackte Wahrheit« bezeichnen könnte. Das Erinnern (an die »Tatsachenwahrheit« selbst) funktioniert aber anders. „Gemeinhin ist Erinnern freilich defizitär: voller Lücken und Brüche, geführt von Interpretationen, die ihm die Gegenwart

43 C. Rodley: Lynch, S. 28.

44 M. Bodmer/M. Rothe: Montage, S. 34. 
diktiert und die Vergangenes, wenn nicht verfälschen, so doch verändern. $\aleph^{45}$ In einer Art autobiographischen Retrospektive spult der sich Erinnernde einzelne Szenen seines Lebens vor und zurück, er schneidet sich wie ein Regisseur sein Leben zurecht und fügt eventuell Szenen im nachhinein ein. Freds Versuch, sich an den »Mord« an seiner Frau $\mathrm{zu}$ erinnern scheitert und sein Kopf schmerzt bei dem Versuch sich zu erinnern. Er hat diese Stelle seines Lebens zensiert und aus seinem »Film« geschnitten. Dieses Phänomen an sich ist kein ungewöhnliches: Unser aller Vergessen ist alltäglich und setzt bereits beim Vergessen der kurz nach dem Aufwachen verschwundenen und doch das Bewusstsein des Menschen maßgeblich bestimmenden Träume ein. Eine Einschränkung des Gedächtnisses erfährt die Leistung des Gedächtnisses überdies durch eben die vorhin beschriebene, natürliche Zensur, die den Menschen vergessen lässt, was ihm unangenehm ist. In diese Lücken des Gedächtnisses kann so die Einbildungskraft eintreten. Denn da, wo die mimetische Kraft des Gedächtnisses versagt, tut sich ein effektiver Spielraum für die Phantasie auf. Seit Platon wird die Einbildungskraft zur Quelle des Denkens, denn ohne Imagination, die Fähigkeit der Einbildungskraft, ist Denken gar nicht möglich. »Die Imagination ist also Funktion des Gedächtnisses, der Retention dessen, was sich ihm eingearbeitet hat, oder sie produziert, dann nämlich, wenn sie die Phantasmen wiederbearbeitet, die sich ihr darbieten, und so hat man ihr seit der Antike den Namen »Phantasie« gegeben. $\ll^{46}$

Die Bedeutungen der Worte »Phantasie« und »Imagination« reichen dabei von Abbildung als »mentalem Bild«, bis zur Einbildung als »Erfindung« oder gar als »Illusion«. Der platonische Begriff der phantasia meint dabei sowohl die wahrheitsgemäße Abbildung als auch die illusorische Erscheinung. Die Erinnerung entwirft folglich eine Gegenwart, die sich immer in den Spuren der Vergangenheit bewegt. »Ich erinnere mich. Dann erinnere ich mich nicht nur einer Vergangenheit,

45 M. Theunissen, Reichweite und Grenzen der Erinnerung, Tübingen 2001, S.17.

46 H. Friese: »Bilder der Erinnerung «, in: M. Neumann (Hg.), Erzählte Identitäten. Ein interdisziplinäres Symposion, München 2000, S. 187 201, hier S. 200. 
sondern auch meiner immer offenen Möglichkeit. « ${ }^{47}$ Diese Imaginationskraft ist folglich nicht bloßer Lückenfüller für eine fortlaufende Geschichte, sondern ein ästhetischer Prozess. Diesen ästhetischen Trick nutzt auch David Lynch, wenn er das »Vergessen« Freds/Petes visuell als Verwandlung darstellt. Letztendlich ist die Imagination Freds/Petes dann der vieldiskutierte Verwandlungsprozess, den das lückenhafte Gedächtnis des Protagonisten hervorbringt und der in der Erzählstruktur den Möbiusschen Kreuzungspunkt markiert.

\title{
5.2 Erinnerung als Verwandlung: Traum oder Realität
}

\author{
»Seit meinem zehnten Lebensjahr, ist es \\ eine Art Glaubenssatz von mir, \\ dass ich aus vielen Personen bestehe, \\ deren ich mir keineswegs bewußt bin. $\ll^{48}$
}

In Lost Highway wird die Erinnerung wörtlich genommen: als Er-Innerung: Gemeint ist hier ein Prozeß, der schon durch den Bindestrich signalisiert wird, »ein Aneignungsproze $\beta$, der auf Selbstaneignung zielt. Wer in sich geht, eignet sich an, als was er sich selbst vorfindet. [...] Mithin vollzieht er einen produktiven Akt, nicht wie der sich Wiedererinnernde einen reproduktiven. Sich aneignend verwandelt er sich zugleich. « ${ }^{49}$ Diese Verschmelzung von Erinnerung und Verwandlung ist nicht neu. Elias Canettis Theorie der Verwandlung entspricht dem Lynchschen Verwandlungsbegriff des Filmes Lost Highway. Nach Canetti kann nur eine Verwandlung im extremen Sinn den wahrhaften Bestand eines Menschen hervorbringen. Positiverweise erreicht man diesen Zustand durch kreative Erinnerung, indem man sich in den Menschen von einst zurückverwandelt. Dass diese positive Verwandlung nicht immer gelingen kann, zeigt er in Masse und Macht. Canetti sagt darin, »Verwandlungen zur Flucht, um einem Feind zu entkommen, sind allgemein. [...] Als die beiden Hauptformen unterscheide ich die lineare von der zirkulären Verwandlungsflucht. ${ }^{50}$

\section{Ebd. S. 201.}

48 E. Canetti: Die gerettete Zunge, Frankfurt/Main 1993, S. 106.

49 M. Theunissen: Reichweite, S. $29 \mathrm{ff}$.

50 E. Canetti: Masse und Macht, Frankfurt/Main 1980, S. 407. 
Die zirkuläre Verwandlung erklärt er mit einer psychischen Krankheit, der Hysterie. Auch Freds/Petes Zustand ist in vielen Rezensionen mit einer Art psychischen Krankheit erklärt worden. Sein Verhalten in der Zelle erweist sich auch als zirkulär, denn er kann nicht aus seiner Haut heraus, er kann nur »die andere Seite in sich« hervorbringen und verwandelt sich so in die neue Figur Pete, die der Zuschauer nun auf der Leinwand sieht. Nur so ist es zu verstehen, dass Pete keineswegs ein Traum oder eine Vision ist. Pete ist die andere Fahrbahn auf dem Möbiusband der Identität. In Canettis Terminologie lautet diese Formel der brüchigen Erinnerung und versuchten Verwandlung: »Ich will mich so lange zerbrechen, bis ich ganz bin. ${ }^{51}$ Die individuelle Wahrnehmung und Erinnerung verlangt also zugleich auch die individuelle Bruchstelle und Verwandlung. Diese extreme schizophrene Erfahrung wird von David Lynch ästhetisch codiert, sie ist für den Zuschauer allerdings nicht komplett verschlüsselt und unzugänglich. Die Einheit von Pete und Fred ist an mehreren Stellen des Films zu erkennen. Den deutlichsten Hinweis darauf liefert das verbindende Element der Jazzmusik, die man in Analogie zu den Videotapes als »auditive Metaphern « fassen könnte. Dieses Element existiert in beiden Figuren. Doch während die Musik für Fred ein Element der Befreiung darstellt, ist sie für Pete, der sie im Radio der Autowerkstatt hört, ein penetranter Stich, der Kopfschmerzen verursacht. Jene Kopfschmerzen, die unterdrückte Erinnerung symbolisieren, verbinden Fred und Pete unzertrennlich und unendlich.

Diese filmische Besonderheit erinnert wiederum an das unendliche Möbiusband. Dessen bekannte, zunächst überraschenden Eigenschaften, z.B. dass man nach dem längsseitigen Zerschneiden eines Möbiusbandes nicht zwei Teile in der Hand hält, sondern nur ein längeres Band, welches bei erneutem Zerschneiden in zwei verkettete Möbiusbänder zerfällt, illustriert die Identität der Figuren Fred/Pete und Renee/Alice auf das deutlichste.

51 E. Canetti: Die Provinz des Menschen: Aufzeichnungen 1942-1972, Frankfurt/Main 1976, S. 180. 


\subsection{Identitätskrise: Identität als Prozess}

»Die Welt, in der wir leben, ist eine Welt der Gegensätze. Der Trick ist, die Gegensätze zu versöhnen. ${ }^{52}$

Identität im allgemeinen bezeichnet die als unabhängig erlebte Einheit des Selbst, die Menschen mit gesunder und reifer Persönlichkeit als selbstverständlich empfinden. Der Psychoanalytiker Erik H. Erikson hat für dieses Stadium den Begriff der Ich-Identität (ego identity) ${ }^{53}$ geprägt. Die gesunde Ich-Identität ermöglicht das funktionierende Handeln gegenüber anderen Menschen, deren Erwartungen ihre Voraussetzungen und ihr Korrelat sind. Sie entsteht durch die verschiedenen Sozialisationen (also einer Reihe von angenommenen und wieder verworfenen Identifikationen), die Kinder bis zum Erwachsenwerden durchleben. ${ }^{54}$

Ein Mensch mit einer gesunden Identität kann sich einerseits Gruppen zugehörig fühlen, ist sich andererseits aber stets bewusst, dass er ein unabhängiges Individuum ist. ${ }^{55}$ Wird der selbstverständliche Prozess der Identität gestört, wie dies bei Fred/Pete der Fall ist, kann im schlimmsten Fall ein Identitätsverlust eintreten, so dass Interaktionen mit der Umwelt nicht mehr gelingen. Als Folge können Störungen der Persönlichkeit wie zum Beispiel Schizophrenie auftreten. ${ }^{56}$ Gerade ein Gedächtnisverlust kann eine funktionierende IchIdentität vehement stören, denn »das Gedächtnis verbindet uns mit Anderen, anstatt uns in unsere Ipseität einzuschließen und uns darauf zu begrenzen. ${ }^{57}$

52 C. Rodley: Lynch, S. 40.

53 Vgl. E.H. Erikson: Identität und Lebenszyklus, Frankfurt/Main 1998, S. 1-212.

54 Vgl. H. Dubiel: »Identität, Ich-Identität«In: J. Ritter/K. Grunder (Hg.), Historisches Wörterbuch der Philosophie, Darmstadt 1976, Bd. 4, S. 218.

55 Ebd., S. 148.

56 Vgl. L. Krappmann: Soziologische Dimension der Identität, Stuttgart 1975, S. 176-178.

57 E.S. Casey: »Erinnerung an den Ort des Anderen«. in: B. Waldenfels/I. Därmann (Hg.), Der Anspruch des Anderen. Perspektiven phänomenologischer Ethik, München 1998, S. 291-316, hier S. 295. 
Jürgen Habermas stellt eine Verbindung zwischen persönlicher Identität, die die unabhängige Person festlegt, und sozialer Identität, die die Gruppenzugehörigkeit des einzelnen manifestiert, her: Von hier aus gesehen stellt sich auch die Identität des Ich, als ein dialogisches Verhältnis dar »In der retrospektiven Deutung des Lebenslaufes kommuniziert das Ich mit sich als seinem Anderen. Selbstbewußtsein konstituiert sich im Schnittpunkt der horizontalen Ebene intersubjektiver Verständigung mit Anderen und der vertikalen Ebene intrasubjektiver Verständigung mit sich selber. $\ll^{58}$ Dieser stille Dialog mit sich selbst bildet auch den Grundstein jener Identitätskrise von Fred/Pete, die in einer extremen psychotischen Störung gipfelt und die man als "psychogene Fuge« bezeichnen könnte. So dient die, im Vorspann hervorstechend gelb gefilmte Mittellinie der Straße als Symbol für den inneren Zustand dieser Hauptfigur. Genau wie sie zwei Fahrbahnhälften voneinander trennt, verhält es sich mit seinem Geisteszustand: er verkörpert zwei Identitätshälften, die nicht strikt von einander getrennt sind. Denn auf der Straße ist die Mittellinie immer durchgängig. Somit besteht die Möglichkeit, dass Elemente der einen Seite in die andere dringen.

"Die Gewohnheit ist es, die uns die Veränderung abstrakt macht, indem diese so langsam vor sich geht, daß wir, indem wir unmerklich ihren Prozeß miterleben, kaum mehr auf sie achtgeben, und es so scheint, als bleibe sich alles gleich. Es bedarf eines Einbruchs des Ungewohnten, [...] damit wir auf den Gegensatz aufmerksam werden, die Tiefe der Schlucht zwischen Veränderung und Gleichheit erkennen. « ${ }^{59}$

Denn, wenn die Gewohnheiten zerbrochen sind und wir auf den Gegensatz aufmerksam werden, verkehren sich Wahrheit und Illusion; was wir für wahr gehalten haben in unserer Gewohnheit, die Gleichheit der anderen, stellt sich als Illusion heraus - der schöne Widerschein wird zum defigurierten Zerrbild transformiert. Dem Identitäts-

58 J. Habermas: Erkenntnis und Interesse, Frankfurt/Main 1968, S.199.

59 C. Hoffmann: Die Konstitution der Ich-Welt. Untersuchung zum Strukturzusammenhang von persönlicher Identität und autobiographischem Schreiben, Würzburg 2000, S. 11. 
suchenden bietet sich so ein Bild des Grauens, dem er sich nur durch Flucht in eine lügenhafte Scheinwelt entziehen kann.

\section{Per Autopilot in den Endlos-Stau}

\subsection{Selbstsuche im Tunnelblick: zwischen Eskapismus und Selbstlüge}

»It's the brain the thing that traps us and ultimately frees you. ${ }^{60}$

Wie vorheriger Abschnitt deutlich machte, stellen die Identitätsproblematik und die daraus resultierende Realitätsflucht die zentrale Thematik des Filmes dar. So führen Freds/Petes gestörte Selbstwahrnehmung und sein ergebnisloser Blick in den Spiegel nicht selten zu dem Wunsch nach Kompensation. Diese kann in Form von Eskapismus und/oder der Selbstlüge erfolgen, wobei letztere mitunter auch die Dimension einer Lebenslüge annehmen kann. Bei der Konstruktion eines solchen parallelen Universums ist es nicht nur schwer, Wunsch und Wahrnehmung voneinander abzugrenzen sondern auch die Verankerung des Ichs in der Welt festzumachen. Daher mündet der Lustgewinn der Selbsttäuschung nicht selten in die Kollision von konstruierter Welt mit anderen Welten. Im Unterschied zur nicht auf sich selbst abzielenden Lüge, liegt das Konfliktpotential nicht so sehr in der Konfrontation mit moralischen Prinzipien oder gesellschaftlichen Normen, sondern findet in der Auseinandersetzung der Innenwelt mit der Außenwelt statt. ${ }^{61}$ In Lost Highway findet dieses Prinzip auch Anwendung, denn keiner der Charaktere ist moralisch integer und frei von Schuld, allen voran die Halbwelt-Figuren, mit denen sich Pete, der ja auch selbst wegen Diebstahls gesucht wird, umgibt. Selbst die vordergründig moralisch angepassten Spießbürger,

60 Vgl. http://www.geocities.com/ mikehartmann/de/index.html vom 21. August 2003.

61 Auch hier lassen sich wieder deutliche Anklänge an den Film noir ausmachen. 
beispielsweise Petes Eltern, handeln sozial verwerflich. Und Fred selbst ist ein mutmaßlicher Mörder, der sich etwas vormacht.

Somit besteht die besondere Leistung der Selbstlüge gerade nicht im Auseinanderhalten sondern vielmehr im Verschwimmenlassen von konstruierter Welt und anderen Welten ${ }^{62}$. Bei Fred/Pete handelt sich allerdings um eine fortgeschrittene Spezialform der Lüge, was in der Psychologie als Pseudologia phantastica, phantastisches Lügen, bezeichnet wird. Dabei glaubt der Lügner selbst an die Wahrheit seiner Behauptung, die nicht selten die Form einer sich verselbständigenden Nebenrealität einnimmt. ${ }^{63}$ Die Verlogenheit ist jedoch nicht nur latent ein zentraler Aspekt im Zusammenhang mit der Identitätsfrage im Film, sondern sie wird auch explizit thematisiert. Beim Showdown in der Hütte - Pete hat sich gerade in Fred zurückverwandelt - fragt dieser den Mystery Man nach Alice. Ungehalten entgegnet der Mystery Man, während er Fred auf Video aufnimmt, folgendes: »Alice who? Her name is Renee. If she's told you her name is Alice she is lying! « Doch dem nicht genug; sofort stellt er Fred die Gegenfrage: »And your name? What the fuck is your name?«, woraufhin Fred wortlos flüchtet. Doch der Mystery Man folgt ihm mit der Kamera und hält sie ihm wie einen Spiegel vor: Doch wer ohne Namen ist, hat auch keine Identität.

\subsection{Der ganz normale Wahnsinn: »Psychogene Fuge«}

\section{»Es ist nicht mehr als ein moralisches Vorurteil,} dass Wahrheit mehr wert ist als Schein. $\ll^{64}$

Lost Highway ist häufig als eine filmische Interpretation einer »Psychogenen Fuge betrachtet worden. Die »Psychogene Fuge Art Bewusstseinsspaltung, die tatsächlich existiert. Sie ist letztendlich eine Form der Amnesie als Flucht vor der Realität. Sie betrifft also eine Person, die ihr ganzes bisheriges Leben verleugnet und in eine neue Identität zu schlüpfen beginnt. Oberflächlich betrachtet trifft dies auch

62 H. Reichert: Lüge und Selbstgespräch, Zwei Kommunikationsmodelle, Wien 1999.

63 Ebd., S. 82.

64 F. Nietzsche: »Jenseits von Gut und Böse«, in: ders., Werke in drei Bänden, Band 3, Köln 1994, S. 6 - 236, hier S. 46. 
auf Fred zu, ließe man oben skizzierte Anmerkung zur Identität als Prozess außer Acht.

Aufschlussreicher als der Begriff »psychogen«, was im Grunde »seelisch bedingt« bedeutet, ist daher der Begriff »Fuge«. Auch wenn dies ein primär musikalischer Begriff ist - und Musik spielt ja eine große Rolle im Film, wie gezeigt wurde - liefert er eine vollständige Beschreibung des Films: ein Thema setzt ein und wird von einem zweiten abgelöst. Das erste Thema läuft begleitend als Kontrapunkt weiter. Eine perfekte Beschreibung der Beziehung zwischen Fred und Pete. Chris Rodley sagt dazu: »Genau, [...] weil es mit einer Sache anfängt, in eine zweite übergeht und dann wie ich meine, an den Anfang zurückkehrt. Genau das ist Lost Highway. ${ }^{65}$ Diese musikalische und seelisch geschlossene Spiralenform ist, wie schon erläutert, vergleichbar mit der Struktur des Möbiusbandes. Zusammenfassend kann man also sagen, dass sich die Elemente Musik, Traum, Erinnerung, Verwandlung und Identität alle auf der Ebene des Möbiusbandes befinden und der Struktur dieses Bandes inhärent sind. Die reale medizinische Form der "psychogenen Fuge« macht nur deutlich, dass der Film seinen Nullpunkt tatsächlich in der Realität haben könnte und nicht »bloß« einen Traum auf Zelluloid bannt.

Lost Highway arbeitet mit hochgradig unkonventionellen Mitteln, um die Problematik von verfahrenen Lügensituationen und verlorenen Identitäten in bestimmten Situationen aufzuzeigen. Weshalb er diese extreme Darstellungsweise gewählt hat, erläutert Lynch folgendermaßen: »Ich interessiere mich für menschliches Verhalten überhaupt. Dafür interessiert sich jeder. Extreme sind irgendwie besonders fesselnd, und Geisteskrankheiten könnten Ideen für etwas auslösen. Durch Geisteskrankheiten verstehen wir vielleicht auch andere Dinge besser. $"{ }^{66}$ Den gewohnten Standpunkt zu verlassen und sich in andere (Denk-)Welten zu begeben ist letztendlich eines der Hauptanliegen von Lost Highway. Wiederum fühlt man sich mit diesem Ansinnen an eine der Lithographien M.C. Eschers (Vgl.: Zauberspiegel, 1946) erinnert und so schließt sich der Kreis um Lost Highway.

65 C. Rodley: Lynch, S. 319.

66 M. Bodmer/M. Rothe: Montage, S. 36. 


\section{Ausfahrt}

》Godard hat gesagt, ein Film brauche einen Anfang, eine Mitte und einen Schluss, aber nicht unbedingt in dieser Reihenfolge. ${ }^{67}$

Abbildung 2: Filmmontage aus Lost Highway

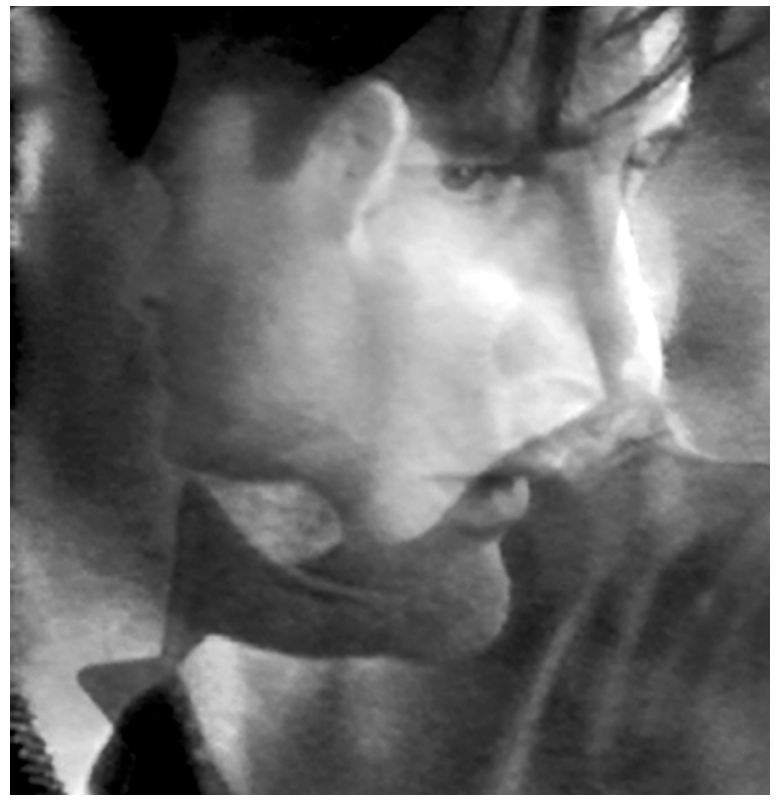

67 David Lynch in: Filmbulletin. Kino in Augenhöhe, 5 (2001), hier S. 1819. 